\title{
POTENCIAL DE TRANSPORTE SEDIMENTAR PELAS CORRENTES DE FUNDO NA REGIÃO DO CANAL DE VEMA (ATLÂNTICO SUL)
}

\author{
Aline Olivas Kajii ${ }^{1}$, Josefa Varela Guerra ${ }^{1}$, Alexandre Macedo Fernandes ${ }^{1}$, Roberto Freires de Oliveira ${ }^{1}$, \\ Cleverson Guizan Silva ${ }^{2}$ e Antonio Tadeu dos Reis ${ }^{1}$
}

Recebido em $1^{\circ}$ março, 2011 / Aceito em 28 junho, 2011

Received on March 1, 2011 / Accepted on June 28, 2011

\begin{abstract}
Vema Channel is an important passageway to the flow of bottom water between the Argentine and the Brazil basins. The channel morphology causes confinement and intensification of bottom currents that affect the ocean floor through sediment resuspension, transport and deposition, consequently playing a fundamental role in the formation of deep ocean deposits. The main goal of this paper is to evaluate the potential for sediment suspension and transport by the local currents through estimates of nearbed shear stresses as well as to assess whether the present dynamic conditions are conducive to the buildup of contouritic deposits in the Vema channel area. The available datasets include (i) current velocity and direction from the World Ocean Circulation Experiment (WOCE) and from moorings deployed by the Woods Hole Oceanographic Institute (WHOI); (ii) hydrographic parameters from the World Ocean Database 2005; and (iii) the description of bottom sediment characteristics and nephelometric profiles available in publications such as research reports and scientific articles. Calculated shear stresses indicate that local currents would be able to erode and transport medium silt with density similar to that of quartz during up to $87 \%$ of the deployment duration, a finding that is reinforced by the nephelometric profiles and by suspended particulate matter concentrations documented by filtered water samples. Channel enlargement downstream leads to Ioss of transport capacity along the channel. These results suggest that a modern drift deposit may still be under construction downstream the channel mouth where a contourite fan has intermittently been built since the late Oligocene.
\end{abstract}

Keywords: Vema Channel, shear velocity, benthic boundary layer, sediment transport.

RESUMO. 0 Canal de Vema é uma importante via de passagem das águas de fundo da Bacia da Argentina para a Bacia do Brasil. A morfologia do canal causa o confinamento e a intensificação das correntes de fundo que têm papel fundamental na ressuspensão, transporte e controle da deposição de sedimentos e consequentemente na formação de depósitos em profundidades abissais. 0 objetivo deste trabalho é avaliar o potencial de ressuspensão e transporte de sedimentos pelas correntes locais através de estimativas do estresse cisalhante junto ao fundo para identificar condições de formação de depósitos contorníticos na região do Canal de Vema. Foram utilizados dados de (i) velocidade e direção das correntes provenientes da base de dados do World Ocean Circulation Experiment (WOCE) e de fundeios realizados pelo Woods Hole Oceanographic Institute (WHOI); (ii) características hidrográficas da região obtidas a partir da base de dados World Ocean Database 2005; e (iii) características granulométricas e perfis nefelométricos descritos na literatura. As estimativas do estresse cisalhante indicam que as correntes no canal são capazes de suspender e transportar silte médio com densidade similar à do quartzo durante até 87\% do tempo. Essa capacidade de ressuspensão de sedimentos também foi observada nos perfis nefelométricos e nas concentrações de MPS total obtidas de amostras de água filtradas. 0 alargamento do canal a jusante leva à redução da capacidade de transporte das correntes. Esses resultados sugerem que um depósito de deriva ainda pode estar em construção a jusante da boca do canal onde um leque contornítico vem sendo construído desde o final do Oligoceno.

Palavras-chave: Canal de Vema, velocidade de cisalhamento, camada-limite de fundo, transporte sedimentar

\footnotetext{
${ }^{1}$ Faculdade de Oceanografia/UERJ, Rua São Francisco Xavier, 524, $4^{\circ}$ andar, Maracanã, 20550-900 Rio de Janeiro, RJ, Brasil. Tel.: (21) 2334-0766 - E-mails: alinekaj@gmail.com; josefa@uerj.br; alexmf@gmail.com; rfreires@gmail.com; tadeu.reis@gmail.com

2Programa de Pós-Graduação em Geologia e Geofísica Marinha, Departamento de Geologia, LAGEMAR, Instituto de Geociências/UFF, Av. General Milton Tavares de Souza, s/n, $4^{\circ}$ andar, Campus da Praia Vermelha, Gragoatá, 24210-346 Niterói, RJ, Brasil. Tel.: (21) 2629-5930/2629-5932; Fax: (21) 2629-5931 - E-mail: cguizan@id.uff.br
} 


\section{INTRODUÇÃo}

0 Canal de Vema está localizado no Atlântico Sul, entre o Platô de São Paulo e a Elevação de Rio Grande (Fig. 1) e, juntamente com o Canal de Hunter, localizado a leste da Elevação de Rio Grande, tem grande importância na evolução do padrão de circulação do Oceano Atlântico, pois desde o Mioceno é a principal via para o fluxo de águas de fundo da Bacia da Argentina para a Bacia do Brasil (Kennett, 1982).

A topografia da região causa 0 confinamento das correntes de fundo e, consequentemente, o aumento de sua intensidade. As correntes exercem papel fundamental no transporte e ressuspensão de sedimentos; dessa forma, espera-se um vigoroso transporte sedimentar ao longo do canal que pode ter gerado depósitos a jusante, onde cessa a intensificação gerada pelo confinamento das correntes. Mézerais et al. (1993) identificaram uma acumulação sedimentar em forma de cone localizada na saída do Canal de Vema, que consiste de contornitos lamosos associados a depósitos de manganês.

Contornitos são sedimentos depositados ou significantemente afetados por correntes de contorno (Rebesco, 2005). Os depósitos contorníticos em águas profundas têm grande importância econômica e científica. A indústria do petróleo é 0 campo que concentra a maior parte dos debates relacionados ao interesse econômico do estudo dos contornitos (Viana, 2008), pois a ação das correntes de contorno pode influenciar a geometria e qualidade dos reservatórios e a distribuição das rochas seladoras (Viana et al., 2007). 0 estudo dos contornitos também é fundamental para entender 0 histórico do clima e circulação dos oceanos e o seu papel na evolução da Terra, pois permitem uma reconstrução paleoceanográfica de alta resolução ao longo das passagens das principais correntes oceânicas (Knutz, 2008). Segundo Rebesco \& Stow (2001), ao decifrar as assinaturas sutis das correntes oceânicas profundas que formaram os depósitos contorníticos é possível encontrar evidências sobre variações de longo prazo nos padrões de paleocirculação e, consequentemente, no paleoclima.

Entretanto, apesar da importância dos depósitos contorníticos e da relevância geológica e paleoceanográfica da região de estudo, os dados disponíveis são escassos. Além disso, há pouco conhecimento sobre os processos sedimentares e oceanográficos em regiões abissais. 0 projeto HEBBLE - "High Energy Benthic Boundary Layer Experiment" (Nowell \& Hollister, 1985) foi pioneiro no estudo do transporte de sedimentos pelas correntes abissais e teve como objetivo entender as respostas dos sedimentos a fluxos de alta energia em regiões abissais, bem como fazer previsões sobre o transporte de sedimentos e respostas do assoalho oceânico a esses eventos. Os resultados desse projeto contribuíram para 0 entendimento da camada-limite de fundo em regiões abissais, e serviram de base para os modelos que são utilizados até hoje.

Neste trabalho foi realizada a reanálise de dados pretéritos sobre a intensidade e a direção das correntes, cobertura sedimentar e hidrografia da região do Canal de Vema e Elevação de Rio Grande para caracterizar a camada-limite de fundo e discutir os possíveis padrões de transporte sedimentar ao longo do canal, a fim de identificar condições para formação de depósitos contorníticos na região.

\section{Camada-limite de fundo}

A camada-limite de fundo, ou camada-limite bentônica (BBL, de Benthic Boundary Layer) é a interface entre o fundo oceânico e a camada d'água sobrejacente onde a fricção do fundo reduz a velocidade do fluido e aumenta a mistura turbulenta (Stahr \& Sanford, 1999), podendo estender-se a até dezenas de metros acima do fundo (Gust, 1989). É através dessa camada que ocorre a transferência de propriedades físicas, químicas e biológicas entre 0 fundo e a coluna d'água. A camada-limite de fundo tem fortes implicações na dissipação de energia produzida pelas correntes oceânicas de larga escala (Salon et al., 2008). Análises da dinâmica da camada-limite de fundo são importantes para obter estimativas sobre a velocidade de fricção e 0 estresse cisalhante do fundo, que são de grande relevância para 0 entendimento dos processos sedimentares.

Lueck (2001) considera que a camada-limite de fundo consiste em três camadas diferentes: (1) uma camada de fundo de Ekman na qual a força de Coriolis, e a fricção turbulenta são importantes; (2) uma camada viscosa bastante fina (milímetros de espessura) próxima ao limite com o substrato, onde a fricção molecular é bastante significante; e (3) uma camada "logarítmica" de transição entre as duas camadas mencionadas, onde a fricção turbulenta prevalece sobre as outras forças e está em equilíbrio com 0 gradiente de pressão.

Segundo Armi \& Millard (1976) a espessura da camada de Ekman de fundo $\left(h_{e}\right)$ pode ser obtida pela relação:

$$
h_{e}=0.4\left(u_{*} / f\right)
$$

onde $u_{*}$ é a velocidade de friç̧ão (ou cisalhante) expressa em $\mathrm{m} / \mathrm{s}$ e $f$ é o parâmetro de Coriolis $(f=2 \Omega \operatorname{sen} \varphi$ ) onde $\Omega$ é a velocidade angular de rotação da Terra em rad/s e $\varphi$ é a latitude. A velocidade de fricção é uma característica do fluxo e é basi- 


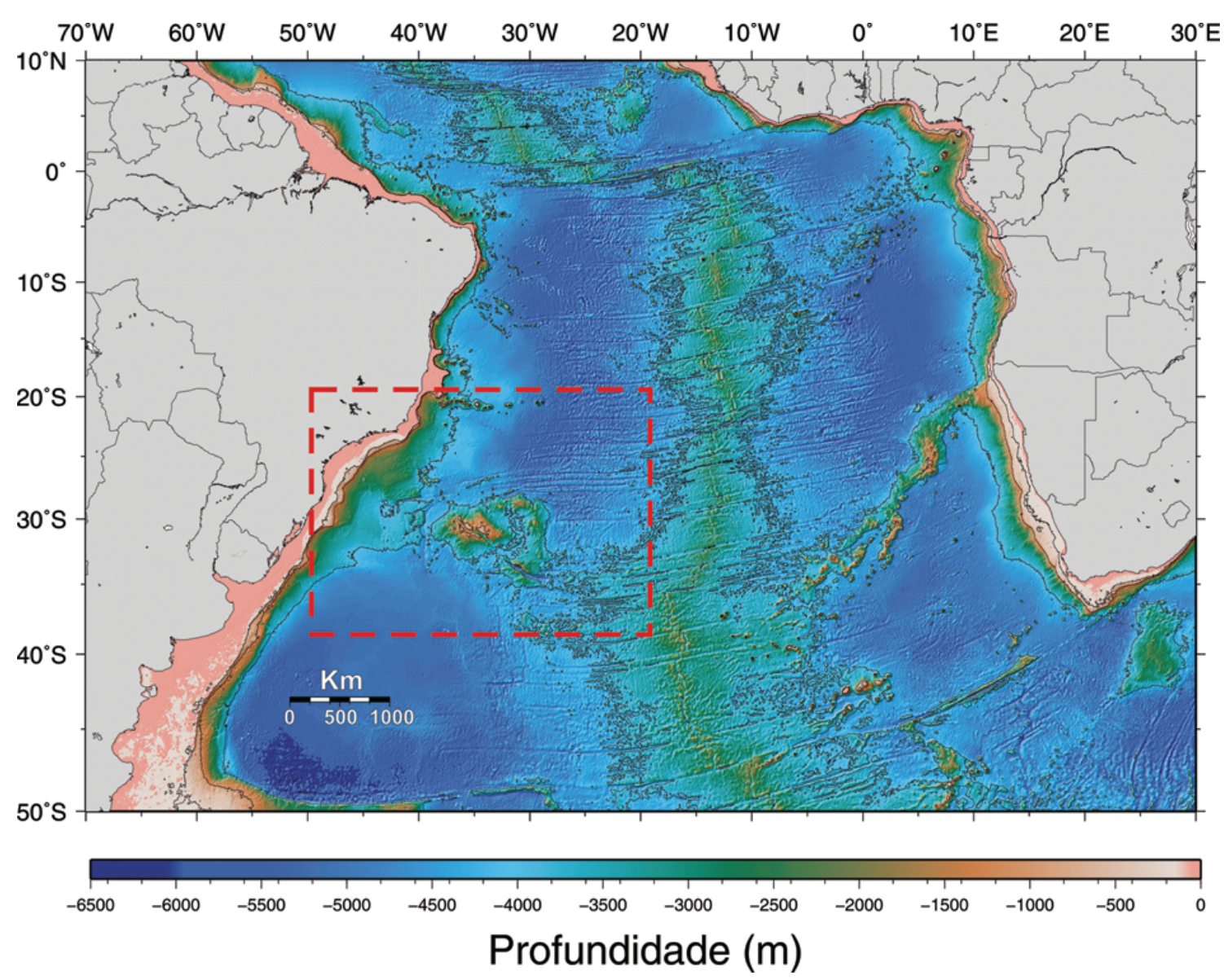

Figura 1 - Localização da área de estudo. Dados batimétricos retirados da base de dados General Bathymetric Chart of the Oceans - GEBCO_08 (GEBC0, 2008) com resolução de $1 / 2$ minuto de grau (Smith \& Sandwell, 1997).

camente determinada pelas flutuações turbulentas da velocidade das correntes na região próxima ao fundo (Salon et al., 2008).

Bem próximo ao fundo há a subcamada viscosa na qual 0 fluxo é lento e dominado pela viscosidade. Essa camada é muito fina, chegando a ter apenas $1 \mathrm{~mm}$ de espessura para um fluxo que move areia muito fina; entretanto, 0 cisalhamento através dessa camada é grande (McCave, 2008). Ainda na camada dominada pela viscosidade há uma zona de transição para a camada turbulenta, uma camada-tampão (buffer layer) (McCave, 2008). 0 modelo de Ekman não é adequado para a subcamada viscosa, pois é necessário levar em conta as irregularidades do assoalho oceânico (rugosidade). Nessa camada, a viscosidade cinemática expressa $\mathrm{em} \mathrm{m}^{2} / \mathrm{s}(v)$ e a velocidade de fricção $\left(u_{*}\right)$ são as únicas grandezas importantes e definem uma escala dimensional, que é a espessura da camada viscosa ( $\left.\delta=v / u_{\downarrow} *\right)$ (Lueck, 2001).

A turbulência na BBL induz a formação de uma camada de mistura de fundo (BML, de Bottom Mixed Layer) que se distin- gue pela homogeneidade vertical das propriedades como temperatura potencial, salinidade e turbidez (Armi \& Millard, 1976; Beaulieu \& Baldwin, 1998). Armi \& Millard (1976) sugerem que a BML é uma feição dinâmica com espessura aproximadamente proporcional à velocidade média diária e correspondente a cerca de seis vezes a espessura da BBL.

As observações do projeto HEBBLE mostraram que a BBL é bastante variável, com um papel importante na mistura da coluna d'água, o que deve ser levado em consideração nos estudos sobre processos sedimentares (Hollister \& Nowell, 1991). As características dos sedimentos também afetam a BBL, pois altas concentrações de sedimentos em suspensão podem alterar as características do campo de densidade, introduzindo estratificação dentro da coluna d'água e, consequentemente, influenciando a dinâmica da camada-limite de fundo (Salon et al., 2008). Além disso, as correntes de fundo são sensíveis à rugosidade do fundo, determinada entre outros fatores, pela presença de formas de fundo, tamanho e forma dos sedimentos. 


\section{Transporte sedimentar e selecionamento}

As circunstâncias necessárias para iniciar o movimento dos sedimentos são função das características do sedimento (densidade, tamanho, selecionamento, forma, entre outras), do fluido (densidade e viscosidade) e das condições do fluxo (velocidade média ou intensidade do estresse turbulento) (Miller et al., 1977).

Li \& Amos (2001) utilizaram o método de Yalin (Miller et al., 1977) para obter 0 parâmetro de Shields $\left(\phi_{c r}\right)$, utilizado para determinar 0 estresse crítico cisalhante para remobilização dos sedimentos:

$$
\begin{aligned}
& \log \theta_{c r}= 0.041(\log Y)^{2}-0.356 \log Y-0.977, \\
& Y<100 \\
& \log \theta_{c r}= 0.132 \log Y-1.804, \\
& 100<Y \leq 3000 \\
& \theta_{c r}=0.045, \quad Y>3000
\end{aligned}
$$

onde o parâmetro adimensional de Yalin é:

$$
Y=\left[\left(\rho_{s}-\rho\right) g D^{3} / \rho v^{2}\right]^{0.5}
$$

onde $\rho_{s}$ é a densidade do sedimento, $\rho$ é a densidade do fluido, ambas expressas em $\mathrm{kg} / \mathrm{m}^{3}$, $v$ é a viscosidade cinemática do fluido e $D$ é 0 diâmetro médio do sedimento expresso em metros.

0 valor de $\phi_{c r}$ pode então ser usado para calcular 0 estresse cisalhante crítico $\left(\tau_{c r}, \mathrm{~N} / \mathrm{m}^{2}\right)$, que é o estresse mínimo necessário para remobilizar partículas sedimentares de determinado tamanho e densidade:

$$
\tau_{c r}=\theta_{c r}\left(\rho_{s}-\rho\right) g D
$$

A velocidade cisalhante crítica $\left(u_{* c r}\right)$ é obtida pela lei quadrática, que relaciona 0 estresse cisalhante crítico com 0 quadrado da velocidade cisalhante crítica:

$$
\tau_{c r}=\rho u_{* c r}^{2}
$$

0 transporte de sedimentos pode se dar junto ao fundo ou em suspensão. Segundo Li \& Amos (2001), o estresse cisalhante crítico para o transporte em suspensão ( $\tau_{c r s}$ ) pode ser calculado a partir da relação:

$$
\tau_{c r s}=0.64 \rho \omega_{s}^{2}
$$

onde $\omega_{S}$ é a velocidade de decantação do sedimento $\mathrm{em} \mathrm{m} / \mathrm{s}$.

0 tipo de transporte é determinado pela relação entre 0 estresse cisalhante gerado pelo fluxo junto ao fundo $\left(\tau_{b}\right)$ e os estresses cisalhantes críticos para 0 transporte junto ao fundo $\left(\tau_{\downarrow} c r\right)$, para 0 transporte em suspensão $\left(\tau_{\downarrow} c r s\right)$ e para deposição $\left(\tau_{d}\right)$. Como 0 valor de $\tau_{d}$ não é bem conhecido, McCave \& Swift (1976) assumem que $\tau_{d}=\tau_{c r}$ para sedimentos nãocoesivos, já que abaixo desse valor 0 deslocamento horizontal cessa e os grãos começam a decantar.

Segundo Bird et al. (1982) a velocidade cisalhante pode ser estimada como sendo 3 a $5 \%$ da velocidade geostrófica no local $\left(U_{G}\right)$ :

$$
u_{* c} \approx(0,03-0,05) U_{G}
$$

Os depósitos contorníticos podem fornecer indicações sobre a velocidade de paleocorrentes através de indicadores como 0 silte selecionável (McCave et al., 1995). 0 transporte, deposição e erosão dos sedimentos pelas correntes de fundo moldam a distribuição granulométrica, que fica registrada nesses depósitos.

\section{ÁREA DE ESTUDO \\ Contexto morfológico}

0 Canal de Vema está localizado entre o Platô de São Paulo e a Elevação de Rio Grande (Fig. 1) e tem entre $25-50$ km de largura e centenas de quilômetros de extensão; sua orientação é controlada, em algum grau, pelo relevo do embasamento acústico abaixo do fundo do canal (Johnson, 1984). A parte mais profunda do canal tem cerca de 4800 metros de profundidade.

Segundo Le Pichon et al. (1971) o Canal de Vema é uma feição erosiva. Perfis de sísmica de reflexão demonstraram que 0 relevo do embasamento subjacente ao canal é bastante irregular, e que essas irregularidades podem ter influenciado a orientação e morfologia do canal durante o seu desenvolvimento. Seções transversais ao longo do canal mostram que 0 canal principal é assimétrico; sua parte mais profunda é em geral adjacente à parede oeste, que tem gradiente acentuado e que, em muitas seções transversais, apresentou 0 afloramento do embasamento acústico. A parede leste do canal se apresenta íngreme até a profundidade de $4200 \mathrm{~m}$ onde há um extenso terraço coberto por ondas de sedimentos (Johnson, 1984). A margem leste do terraço se funde com os flancos mais baixos da Elevação de Rio Grande (Le Pichon et al., 1971). Próximo à latitude de $30^{\circ} 40^{\prime} \mathrm{S}$ o canal apresenta uma bifurcação na qual um canal secundário diverge para oeste (Johnson et al., 1976).

No fundo do canal principal, o sedimento superficial apresenta grande quantidade de minerais de argila misturados com outros minerais (quartzo e mica muito finos), fósseis silicosos, detritos de peixes, foraminíferos bentônicos, nódulos de manganês, vidro vulcânico, grãos de óxido de ferro e raros traços de fósseis calcários (Melguen \& Thiede, 1974). Na parede oeste do canal, mais escarpada, também não foi encontrado carbonato; 


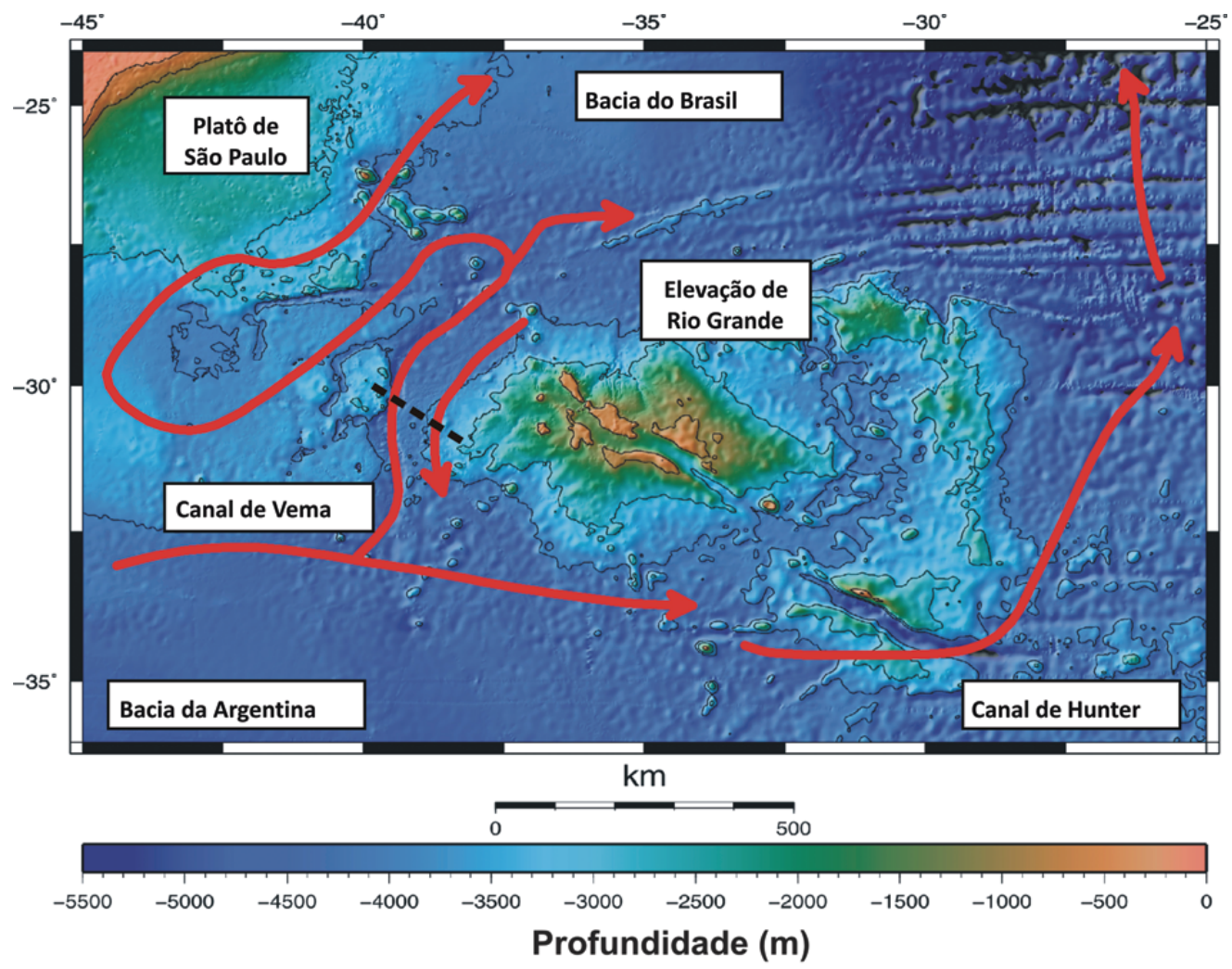

Figura 2 - Padrão esquemático do fluxo de AABW na região, baseado em McDonagh et al. (2002) e Morozov et al. (2008). A linha pontilhada indica a localização do perfil mostrado na Figura 3.

entretanto, na parede leste, de declive mais suave, a cobertura sedimentar consiste de lama calcária a vasas calcárias (Johnson et al., 1977). Segundo Melguen \& Thiede (1974) há um aumento do conteúdo de carbonato (de $<2 \%$ a $>85 \%$ ) e da contribuição da fração grossa da parte mais profunda do canal ao topo da Elevação de Rio Grande (o percentual da fração grossa variou de $0,5$ a $50 \%)$.

Análises da parte superficial de um testemunho coletado no flanco leste do Canal de Vema mostram que, em geral, a fração areia corresponde a um pequeno percentual da distribuição granulométrica (3\%), sendo que as frações silte e argila representam, respectivamente, $40 \%$ e $57 \%$ do sedimento total (Barker et al., 1983). Análises da fração silte realizadas em amostras superficiais de sedimentos na região mostraram que, a partir de $4000 \mathrm{~m}$, 0 tamanho médio do silte varia entre $0,0156 \mathrm{e}$ $0,0237 \mathrm{~mm}$ no ponto mais profundo (Ledbetter, 1984).

\section{Contexto oceanográfico}

A circulação abissal do Canal de Vema é dominada pela Água Antártica de Fundo (AAF; Antarctic Bottom Water, AABW). Acima dessa massa d'água encontra-se a Água Profunda do Atlântico
Norte (APAN; North Atlantic Deep Water, NADW), a Água Circumpolar Superior (ACS, Upper Circumpolar Deep Water, UCDW) e encerrando as massas d'águas situadas abaixo da termoclina há a Água Intermediária Antártica (AIA; Antarctic Intermediate Water, $A A I W)$. 0 Canal de Vema é a principal passagem de AAF da Bacia da Argentina para a Bacia do Brasil.

A AAF, com temperaturas potenciais abaixo de $2^{\circ} \mathrm{C}$ (correspondente a uma densidade $\sim 45,85 \sigma_{4}$ ) não é uma massa d'água homogênea (Zenk \& Morozov, 2007), podendo ser subdividida em Água Profunda do Mar de Weddell (APMW; Weddell Sea Deep Water, WSDW), uma massa d'água mais densa encontrada junto ao fundo, e Água Circumpolar Inferior (ACl; Lower Circumpolar Deep Water, LCDW) acima, sendo separadas pela isoterma de $0,2^{\circ} \mathrm{C}$. Logo acima, o fluxo dentro da camada ocupada pela APAN é predominantemente dirigido para 0 sul com alguma influência da topografia. Hogg et al. (1999) observaram que há um controle sobre a orientação e aceleração do fluxo sobre o Canal de Vema e a divisão do fluxo para oeste sobre uma depressão no Platô de São Paulo. Essa depressão tem um efeito ainda mais significativo sobre a AAF, causando recirculação da água que entra no canal sobre o platô de São Paulo (Fig. 2). Acima da APAN, há a AIA e a ACS com fluxo para oeste e para 
sul como parte da circulação anticiclônica, subtropical, controlada pelo vento (Hogg et al., 1999).

0 fluxo da AAF é predominantemente dirigido para norte, embora em algumas áreas, como no flanco leste do canal, o fluxo seja para sul (Hogg et al., 1999). McDonagh et al. (2002) observaram que, apesar de menos estratificada, a ACI transportada por esse fluxo para sul tem propriedades de salinidade e densidade potencial semelhantes àquela encontrada na parte principal do Canal de Vema, o que pode significar que 0 fluxo do flanco leste é alimentado pelo fluxo do canal principal ou é o resultado de mistura lateral. Como as mesmas características não foram observadas ao sul da Elevação de Rio Grande McDonagh et al. (2002) acreditam que o fluxo pode ser resultado de uma recirculação local não necessariamente conectada com as bacias adjacentes. Johnson et al. (1976) observaram que também há um transporte substancial de AAF para norte dentro do canal secundário (a oeste), apesar de este ser algumas centenas de metros mais raso que 0 canal principal.

Hogg et al. (1999) calcularam um transporte de AAF de cerca de 4 Sv através do Canal de Vema, semelhante ao transporte que havia sido estimado por Hogg et al. (1982). McDonagh et al. (2002) calcularam valores de 3,7 $\pm 0,7 \mathrm{~Sv}$ dentro do canal principal e transporte de $1,7 \pm 0,6 \mathrm{~Sv}$, para sul no flanco leste do canal. Hogg \& Zenk (1997) identificaram mudanças de longo período no transporte de AAF, associadas a variações na temperatura das águas de fundo. Valores bastante elevados de velocidade das correntes já foram registrados; Zenk (2008) analisou dados de velocidade e direção das correntes dentro do Canal de Vema a 50 metros acima do fundo durante 687 dias e encontrou valores médios de $18,4 \mathrm{~cm} / \mathrm{s}$. 0 valor máximo encontrado para a componente zonal das correntes foi de $35,6 \mathrm{~cm} / \mathrm{s}$; Zenk (2008) verificou que essa componente da corrente excede em aproximadamente oito vezes a componente meridional. Ledbetter \& Johnson (1976), utilizando o tamanho médio do silte da porção sem carbonato do sedimento superficial, definiram uma zona de sedimentos mais grossos que corresponde à área sob influência do fluxo de AAF com as maiores velocidades, localizada adjacente à parede oeste do canal. 0 confinamento gerado pelo canal não tem efeitos somente sobre as correntes; Johnson et al. (1976) verificaram 0 aumento da espessura da camada nefeloide de fundo (de dezenas de metros na Bacia da Argentina para centenas de metros dentro do canal).

\section{Paleoceanografia}

A partir de análise de testemunhos coletados na região do Canal de Vema, Ledbetter (1979) estimou taxas de sedimentação de aproximadamente $1 \mathrm{~cm} / 1000$ anos nas amostras mais rasas que 4100 metros (área de domínio da APAN). As maiores taxas de sedimentação foram encontradas próximo ao limite leste da AAF ( $\sim 4180 \mathrm{~m})$ onde a velocidade é lateralmente reduzida na zona de transição com a APAN (Fig. 3). A extrapolação da tendência de diminuição das taxas de sedimentação a oeste desse máximo sugere que a região abaixo de 4300 metros esteve sujeita a intensa erosão seletiva.

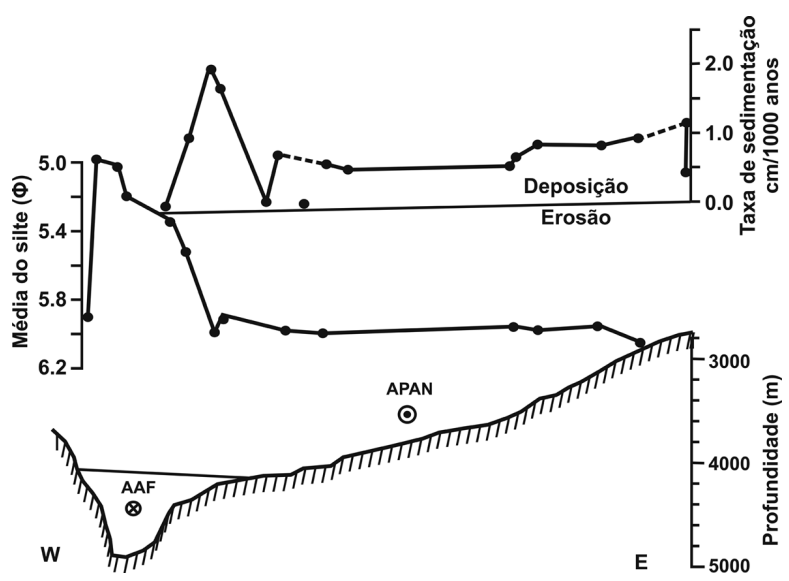

Figura 3 - Embaixo: Perfil batimétrico idealizado do Canal de Vema (localização na Fig. 2). 0 limite entre AABW e NADW corresponde ao ponto médio de menor velocidade. Centro: Tamanho médio do silte sem carbonato (em unidades de $\Phi$ ( $D=D_{0} 2^{-\phi}$, onde $D$ é 0 diâmetro da partícula em $\mathrm{mm}, D_{0}$ é 0 diâmetro de referência $=1 \mathrm{~mm}$ e $\Phi$ é a escala phi)) de amostras superficiais. Os valores devem ser utilizados somente para comparação entre as diferentes regiões do canal. Acima: Taxa de sedimentação. Modificado de Ledbetter (1979).

Com a utilização do tamanho médio do silte sem carbonato como indicador de flutuações de paleovelocidade, Ledbetter (1979) encontrou em todos os testemunhos analisados zonas síncronas de intensa erosão seletiva causada pelo aumento relativo da velocidade das águas de fundo; estas estiveram sempre acima dos valores das velocidades atuais, especialmente durante três períodos: entre 11000-18000, 28000-85000 e 125000-130000 anos A.P. com um aumento menos expressivo há 22000 anos A.P.

\section{METODOLOGIA}

Para a realização deste trabalho foram analisadas séries temporais disponíveis em bases de dados de diversas instituições. Foram realizadas reanálises dos dados de intensidade e direção das correntes provenientes de fundeios realizados entre $1991 \mathrm{e}$ 1992 durante 0 Deep Basin Experiment do World Ocean Circulation Experiment (WOCE), descritos por Hogg et al. (1996; 1999) (VE/338; Fig. 4). Neste artigo foram utilizados os dados de apenas um desses fundeios, no qual foram realizadas medições 


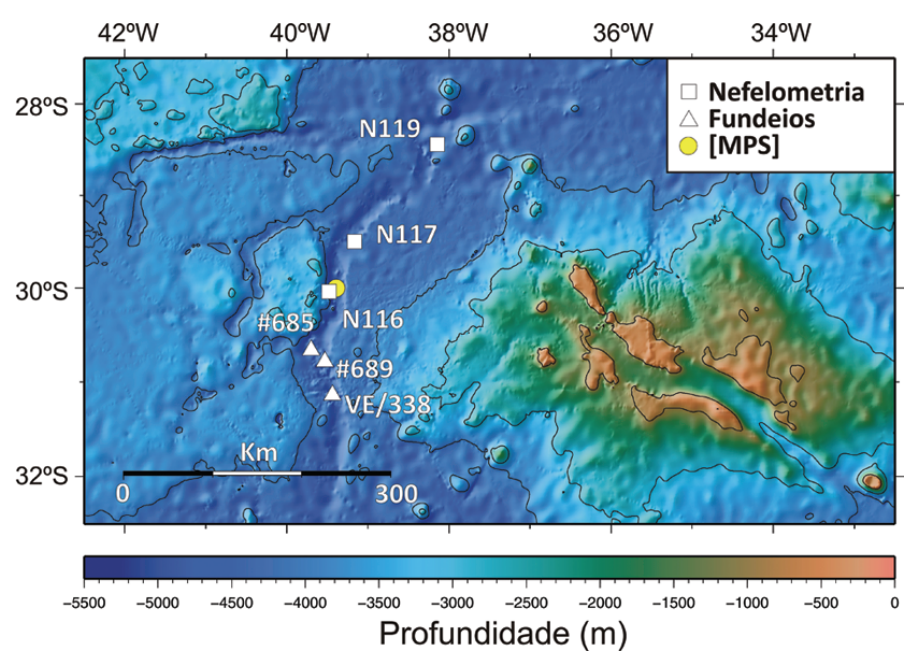

Figura 4 - Mapa de localização dos fundeios $(\Delta)$, perfis de nefelometria $(\square)$ e estação de coleta de MPS $(\bigcirc)$.

a cada duas horas com correntômetros fabricados pela Aanderaa Inc., modelo RCM 8 que são equipados com sensores de temperatura. Também foram utilizados dados provenientes de dois fundeios realizados pelo Woods Hole Oceanographic Institute (WHOI) entre 1980 e 1981 com duração de aproximadamente um ano (Hogg et al., 1982; Schmitz \& Hogg, 1983) (\#685, \#689; Fig. 4); estas medições foram realizadas a cada hora por correntógrafos COS/MOS 850 que também são equipados com sensores de temperatura. Os dados hidrográficos foram obtidos junto à base de dados World Ocean Database 2005 do National Oceanographic Data Center (NODC).

As informações sobre a cobertura sedimentar da região foram obtidas a partir dos relatórios de bordo que descrevem testemunhos coletados durante os cruzeiros dos navios "Atlantis II" 107-9 (1980) e "Chain" 115-6 (1974) (Ledbetter, 1984) e de testemunhos do Deep-Sea Drilling Project (Barker et al., 1983). Os perfis nefelométricos foram obtidos durante 0 cruzeiro "Conrad" 15 em 1972 (Fig. 4); os detalhes sobre o equipamento bem como os dados estão disponíveis em Sullivan et al. (1973). Também foram utilizadas séries temporais de dados de espalhamento da luz do nefelômetro LDGO-Thorndike obtidos simultaneamente aos fundeios de 1980/81 (Richarson et al., 1987).

As séries temporais de velocidade e direção das correntes foram submetidas à análise espectral para identificar as forçantes físicas mais significativas atuantes na região; diagramas T-S foram construídos com os dados hidrográficos para identificação das massas d'água presentes.

Os dados de intensidade das correntes e das características granulométricas foram aplicados em equações, baseadas em teorias sobre a camada-limite de fundo, para que fossem estimadas a velocidade próxima ao fundo, a velocidade crítica de cisalha- mento para ressuspensão de sedimentos e a velocidade de cisalhamento (Eqs. 1 a 9). Os dados de temperatura e salinidade, utilizados para 0 cálculo da viscosidade cinemática, foram obtidos a partir de perfis de CTD realizados durante a instalação dos fundeios.

As velocidades de cisalhamento foram estimadas segundo Bird et al. (1982) (Eq. 9), a partir dos valores de velocidade das correntes medidos a 349, 396 e 250 metros acima do fundo, respectivamente, em estações identificadas como \#685, \#689 e VE/338 (Fig. 4), após a verificação de que o fluxo nessas profundidades era geostrófico. Para esta determinação foram calculados os números adimensionais Rossby temporal $\left(R o_{T}\right)$, de Rossby $(R o)$ e de Ekman $(E k)$ que quantificam a importância de diversos termos em relação ao parâmetro de Coriolis (Cushman-Roisin \& Beckers, 2007; Tab. 2). 0 número de Rossby temporal compara a taxa temporal da mudança da velocidade $(T)$ com a força de Coriolis $\left(F_{0}\right)$ :

$$
R o_{T}=\frac{1}{f_{o} T}
$$

0 número de Rossby compara a advecção com a força de Coriolis:

$$
R o=\frac{U_{G}}{f_{o} \Delta y}
$$

onde $U_{G}$ é velocidade média do fluxo no Canal de Vema e $\Delta y$ é a distância entre os flancos do canal.

0 número de Ekman mede a importância relativa da fricção com o fundo:

$$
E k=\frac{A_{z}}{f_{o} H^{2}}
$$

onde $A_{Z}$ é 0 coeficiente de viscosidade turbulenta e $H$ é a distância ao fundo. 
Tabela 1 - Números de Rossby temporal $\left(R o_{T}\right)$, de Rossby $(R o)$ e de Ekman ( $E k$ ) calculados para cada fundeio. Os valores de $T$ utilizados para o cálculo do número de Rossby temporal foram obtidos através da análise espectral das séries temporais. Para o cálculo do número de Ekman assumiu-se, para uma estimativa conservadora, $A_{z}=10^{-5}$, um dos menores valores encontrados nos oceanos.

\begin{tabular}{|c|c|c|c|c|}
\hline Fundeio & $\begin{array}{c}\text { Distância a0 } \\
\text { fundo (m) }\end{array}$ & $R o_{T}$ & $R o$ & $E k$ \\
\hline$\# 689$ & 396 & $0,0026-0,0048$ & 0,1058 & $<0,0085$ \\
$\# 685$ & 349 & $0,0049-0,0097$ & 0,0480 & $<0,0110$ \\
VE/338 & 250 & $0,0037-0,0111$ & 0,3008 & $<0,0212$ \\
\hline
\end{tabular}

Como os valores calculados (Tab. 1) são pelo menos uma ordem de grandeza menor do que 1 pode-se considerar que 0 fluxo está aproximadamente em equilíbrio geostrófico (CushmanRoisin \& Beckers, 2007).

A partir das velocidades de cisalhamento encontradas, a espessura da camada de Ekman $\left(h_{e}\right)$ foi estimada segundo Armi \& Millard (1976; Eq. 1). Também foram calculadas as velocidades de cisalhamento críticas para início do transporte de sedimentos de diversos tamanhos granulométricos (Eqs. 6, 7 e 8) utilizando o modelo de transporte sedimentar SEDTRANS (Neumeier et al., 2008).

\section{RESULTADOS E DISCUSSÕES}

\section{Correntes}

No fundeio \#689 (1980-81), o correntômetro mais próximo ao fundo foi instalado a 96 metros acima do fundo (maf). Durante 0 período de medição, a intensidade média das correntes foi de $22,4 \mathrm{~cm} / \mathrm{s}$ e houve apenas três eventos em que esses valores foram superiores a $40 \mathrm{~cm} / \mathrm{s}$; por outro lado, apesar da variação temporal observada as correntes raramente atingiram valores abaixo de $10 \mathrm{~cm} / \mathrm{s}$ (Fig. 5a; Tab. 2). As correntes medidas a 396 maf apresentaram comportamento semelhante às observadas na região mais próxima ao fundo, indicando que o fluxo a 96 maf ainda é geostrófico (Fig. 5b). Por estar localizado em um ponto menos confinado (acima do talvegue), o correntômetro a 747 maf registrou os menores valores de velocidade das correntes nesse fundeio (Fig. 5c; Tab. 2).

No fundeio \#685 (1980-81) as correntes medidas mais próximo ao fundo (48 maf) foram menos intensas do que no fundeio \#689, com valor médio de $12 \mathrm{~cm} / \mathrm{s}$ e máximo de $33,8 \mathrm{~cm} / \mathrm{s}$ (Fig. 6a; Tab. 2). A 349 maf as correntes registradas foram mais intensas, com valor médio de $13,4 \mathrm{~cm} / \mathrm{s}$ e máximo de $39,0 \mathrm{~cm} / \mathrm{s}$ (Fig. 6b; Tab. 2), mas ainda assim mais fracas do que no fundeio \#689 (Fig. 5b; Tab. 2). A 704 maf foi observado um padrão similar das correntes medidas a 48 e 349 maf, com valor médio de 10,7 cm/s (Fig. 6c; Tab. 2).
No fundeio VE338 (1991-92) as medições da intensidade e direção das correntes mais próximo ao fundo foram realizadas a 50 maf; 0 maior valor registrado foi de $57,2 \mathrm{~m} / \mathrm{s}$ e a média ao longo de dois anos foi de $30,2 \mathrm{~cm} / \mathrm{s}$ (Fig. 7a; Tab. 2). Neste fundeio apesar de a intensidade das correntes apresentar alguma variação temporal raramente alcançou valores abaixo de $20 \mathrm{~cm} / \mathrm{s}$. Nas medições a 250 maf foram observadas as maiores intensidades entre todos os fundeios analisados, alcançando valores de até $71,1 \mathrm{~cm} / \mathrm{s}$ (Fig. 7b; Tab. 2). 0 correntômetro a 525 maf registrou os menores valores de velocidade das correntes neste fundeio, bem como uma pequena mudança de direção para NW, devido ao menor confinamento nessa profundidade (Fig. 7c; Tab. 2).

Em todos os fundeios analisados as correntes medidas mais próximo ao fundo foram, em geral, unidirecionais, aproximadamente de sul para norte nos fundeios \#689 e \#685 e de SSE para NNW no fundeio VE/338, seguindo a orientação do canal (Figs. 5, 6 e 7). No fundeio \#689 houve um período em agosto de 1980 onde foi observada uma inversão da direção das correntes (Fig. 5). Ao mesmo tempo foi registrado no sensor a 396 maf um aumento brusco na temperatura $\left(\Delta T=+0,6^{\circ} \mathrm{C}\right)$. Hogg et al. (1982) e Johnson (1984) sugerem que essa mudança de direção ocorreu mais provavelmente devido à migração de um vórtice de mesoescala através do canal e não devido a uma interrupção total do fluxo de AAF, já que essa anomalia foi observada em apenas um fundeio (não foram observadas reversões similares no fundeio \#685 durante esse mesmo período).

Tabela 2 - Velocidades médias e máximas das correntes em diversas profundidades dos fundeios \#689, \#685 e VE/338 (localização na Fig. 4).

\begin{tabular}{|c|c|c|c|}
\hline Fundeio & $\begin{array}{c}\text { Distância } \\
\text { ao fundo }(\mathrm{m})\end{array}$ & $\begin{array}{c}\text { Vel. média } \\
\text { (cm/s) }\end{array}$ & $\begin{array}{c}\text { Vel. máx. } \\
\text { (cm/s) }\end{array}$ \\
\hline \multirow{3}{*}{ \#689 } & 747 & 9,8 & 30,7 \\
& 369 & 22,3 & 51,9 \\
& 96 & 22,3 & 51,4 \\
\hline \multirow{3}{*}{ \#685 } & 704 & 10,4 & 39,4 \\
& 349 & 13,4 & 39,0 \\
& 48 & 11,9 & 33,8 \\
\hline \multirow{3}{*}{ VE/338 } & 525 & 18,8 & 64,4 \\
& 250 & 36,3 & 71,1 \\
& 50 & 30,2 & 51,2 \\
\hline
\end{tabular}

A análise espectral das séries de correntes medidas próximo ao fundo dos fundeios \#685, \#689, e VE/338 (Figs. 8, 9 e 10) revela a dominância da componente meridional da velocidade em relação à componente zonal, o que se deve à orientação predominantemente SSE-NNW do canal. A variabilidade associada à maré semidiurna é observada em todas as séries temporais 


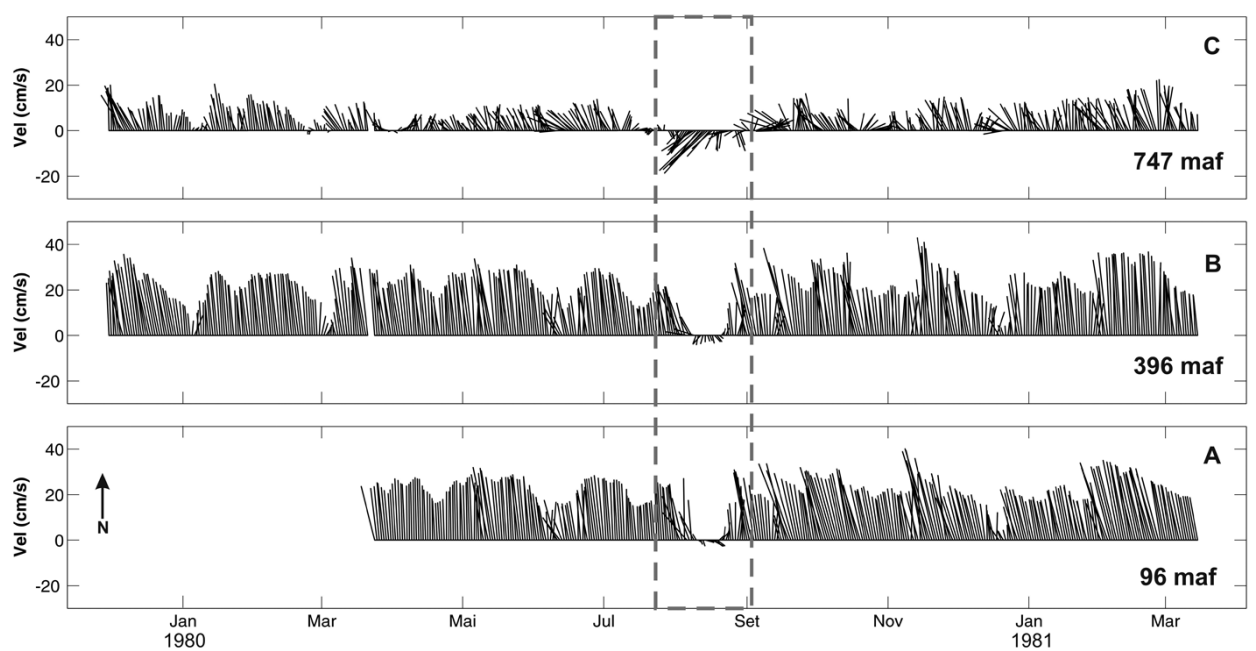

Figura 5 - Médias diárias da intensidade e direção das correntes no fundeio \#689 (A) a 96 maf, (B) a 396 maf e (C) a 747 maf. Profundidade local: $4656 \mathrm{~m}$. Em destaque o período de inversão de direção das correntes, com duração de aproximadamente 10 dias. Dados disponíveis no WHOI Buoy Group Time Series (2010).

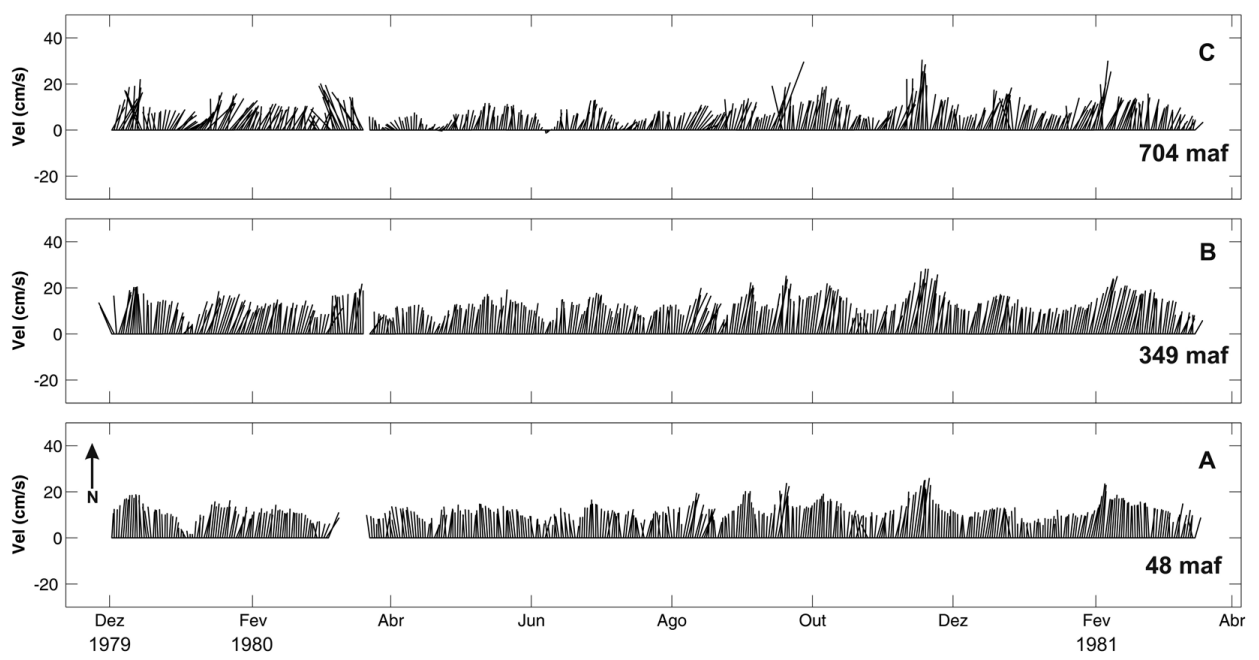

Figura 6 - Médias diárias da intensidade e direção das correntes no fundeio \#685 (A) a 48 maf, (B) a 349 maf e (C) a 704 maf. Profundidade local: 4637 m. Dados disponíveis no WHOI Buoy Group Time Series (2010).

analisadas sendo mais proeminente no fundeio \#685. Este fundeio encontra-se numa área menos confinada do canal onde a componente zonal da velocidade varia mais intensamente sob 0 efeito da maré contribuindo assim para um pico mais energético da velocidade total. Parte da variabilidade das correntes medidas foi observada em períodos de 14-17 dias. Essa variabilidade foi também verificada por Zenk (2008), a partir da análise de séries temporais de corrente e temperatura coletados na coluna d'água a $220 \mathrm{~m}$ acima do fundo, durante os anos de 1998 e 1999, sendo associada à atividade de vórtices de submesoescala. Nas séries de temperatura analisadas nesse estudo 0 autor também detectou colapsos episódicos na estratificação, caracterizados por flutua- ções na profundidade das isotermas de 0 e $0,2^{\circ} \mathrm{C}$. Esses eventos foram observados em períodos de 3-4 semanas e associados à penetração do ramo superior da AAF (LCDW), mais quente, e do ramo inferior (APMW), relativamente mais frio. Os dados analisados no presente trabalho indicam que essa penetração pode ter ocorrido por cerca de 2-3 semanas no mês de agosto de 1980, como observado nos dados do fundeio \#689 pela inversão de fluxo na série de velocidade (Fig. 5) e pelo aumento da temperatura. Como discutido por Zenk (2008), o deslocamento para norte de vórtices abissais formados na Bacia da Argentina, originalmente descritos por Arhan et al. (2002), seria uma possível forçante associada a esses eventos. Segundo Zenk (2008), esses 

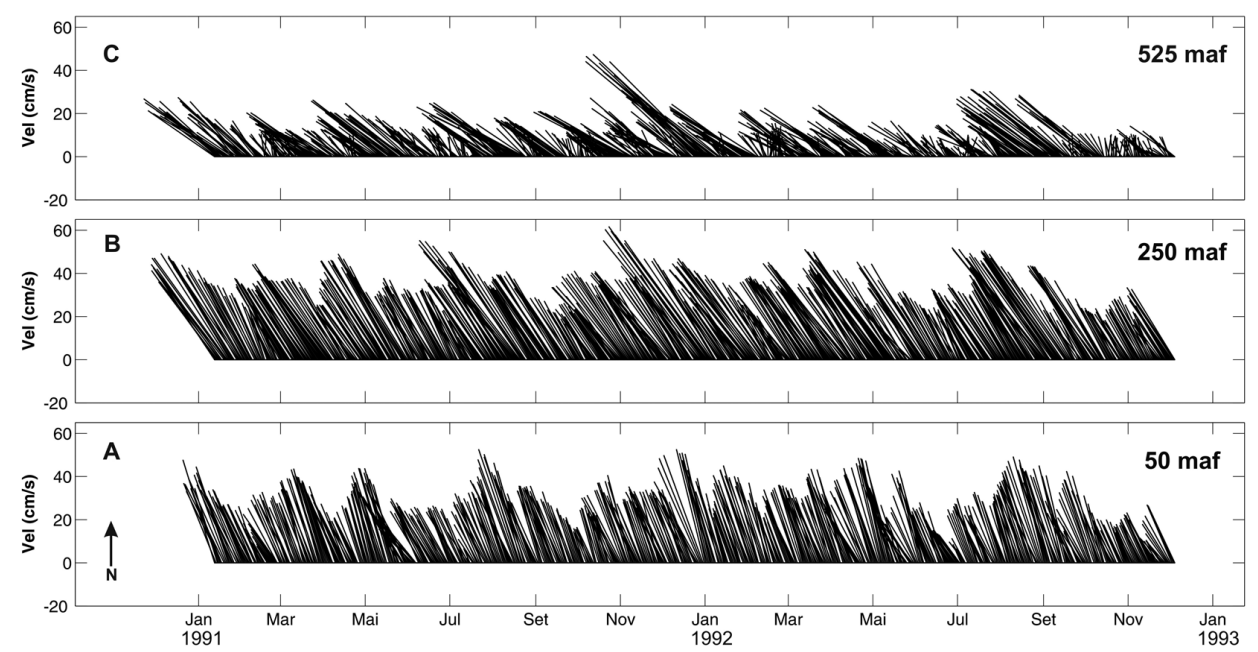

Figura 7 - Médias diárias da intensidade e direção das correntes no fundeio VE/338 (A) a 50 maf, (B) a 250 maf e (C) a 525 maf. Profundidade local: $4675 \mathrm{~m}$. É importante notar a diferença de escala entre os fundeios. Dados disponíveis no WOCE Global Data Resource Version 3.0 (2009).

vórtices seriam atraídos para o Canal de Vema devido ao fluxo afunilado em seu interior, junto ao fundo. 0 evento observado, no mês agosto, sugere a presença de um vórtice anticiclônico.

Além da passagem de vórtices abissais, a ação de longas ondas de Rossby baroclínicas pode ter contribuído para a variabilidade presente nos espectros analisados, em períodos de 35 a 65 dias. De acordo com Zenk (2008), esses mecanismos foram identificados por Lukas et al. (2001) como forçantes atuantes no fluxo através da soleira ( "sil/") entre Maui e Kauai na região subtropical do Pacífico Norte.

Em regimes de mais baixa frequência, a análise espectral, em particular para os fundeios \#685 e VE/338, sugere que uma série de dados mais longa exibiria variabilidade significativa, possivelmente, em escala anual.

\section{Características hidrográficas}

A análise do diagrama T-S espalhado, construído a partir de perfis de temperatura, salinidade e densidade coletados na região durante a instalação dos fundeios, permitiu a identificação das massas d'água presentes no Canal de Vema (Fig. 11): Água Antártica de Fundo (AAF), Água Profunda do Atlântico Norte (APAN), a Água Circumpolar Superior (UCDW) e Água Intermediária Antártica (AAIW). Esses mesmos perfis mostram a presença das águas de origem austral (AAIW e UCDW) nas profundidades intermediárias (águas de baixa salinidade, situadas em torno de 800 a $2000 \mathrm{~m}$ ), seguidas por uma espessa camada de salinidade mais alta, característica da APAN. As últimas centenas de metros da coluna d'água são ocupadas pela Água Antártica de Fundo (AAF) $\left(\mathrm{T}<2^{\circ} \mathrm{C}\right)$.

\section{Camada-limite de fundo}

0 perfil de CTD realizado próximo ao fundeio \#689 mostrou uma camada de mistura de fundo (BML) com espessura de cerca de 300 metros, enquanto a espessura estimada da camada de Ekman de fundo foi de 34 a 56 metros (Tab. 3). Já a espessura da BML no fundeio VE/338 era de cerca de 550 metros, 0 que representa cerca de seis vezes a espessura da camada de Ekman de fundo estimada para esse dia (Fig. 12; Tab. 3), mesma relação constatada anteriormente por Armi \& Millard (1976) na região da planície abissal de Hatteras, no Atlântico Norte. No perfil de CTD do fundeio \#685 essa correlação entre a BML e a camada de Ekman não foi observada, sugerindo que a camada de mistura de fundo nesse local foi formada sob condições mais energéticas a montante e advectada pelas correntes através do canal (Armi \& Millard, 1976).

Tabela 3 - Velocidade média das correntes medidas no dia da realização dos perfis de CTD, profundidade de medição das correntes, espessura estimada da camada de Ekman de fundo no mesmo dia e espessura da camada de mistura de fundo (BML) nos fundeios \#689, \#685 e VE/338. *as medições dessas estações foram realizadas no dia seguinte do perfil de CTD.

\begin{tabular}{|c|c|c|c|c|}
\hline Fundeio & $\begin{array}{c}\text { Vel. média } \\
(\mathrm{cm} / \mathrm{s})\end{array}$ & $\begin{array}{c}\text { Prof. de medição } \\
\text { das correntes } \\
(\mathrm{maf})\end{array}$ & $\begin{array}{c}h_{e} \text { média } \\
(\mathrm{m})\end{array}$ & $\begin{array}{c}\text { Espessura } \\
\text { da BML } \\
(\mathrm{m})\end{array}$ \\
\hline$\# 689$ & 22,8 & 396 & $34-56$ & 300 \\
$\# 685$ & $10,0^{*}$ & 48 & $21-35$ & 270 \\
VE/338 & $48,4^{*}$ & 50 & $73-121$ & 550 \\
\hline
\end{tabular}

Os perfis de temperatura e salinidade do fundeio VE/338 apresentam uma feição em formato de degrau que está associado a uma redução da velocidade média diária. Armi \& Millard 


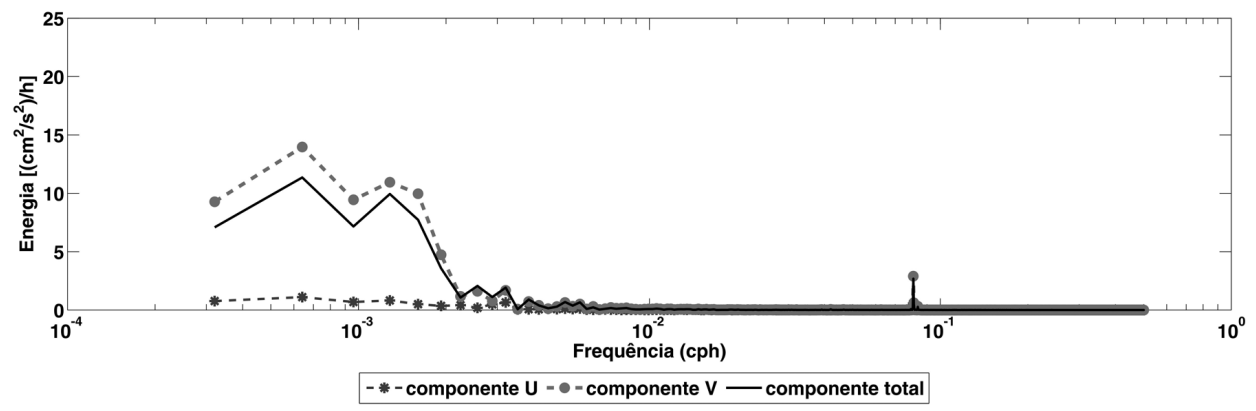

Figura 8 - Análise espectral das correntes medidas a 96 metros acima do fundo no fundeio \#689. Frequência em ciclos por hora. As componentes mais relevantes são de baixa frequência, havendo um pico de maior frequência correspondente à maré semidiurna.

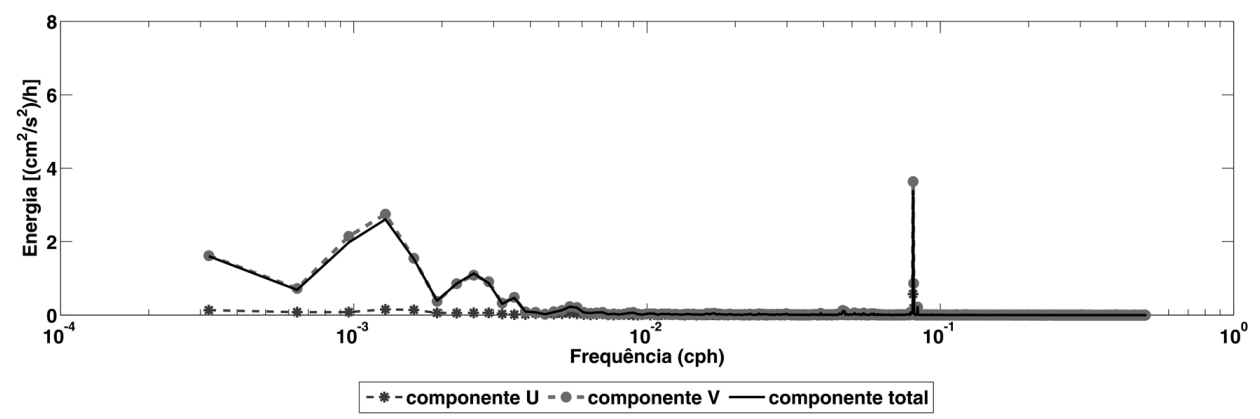

Figura 9 - Análise espectral das correntes medidas a 50 metros acima do fundo no fundeio VE/338. Frequência em ciclos por hora. As componentes mais relevantes são de baixa frequência, havendo um pico de maior frequência correspondente à maré semidiurna.

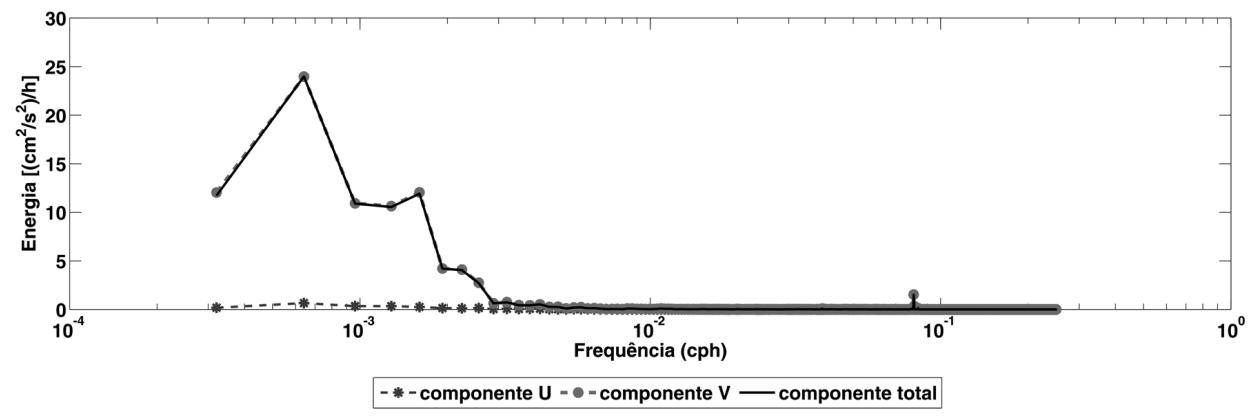

Figura 10 - Análise espectral das correntes medidas a 48 metros acima do fundo no fundeio \#685. Frequência em ciclos por hora. A frequência da maré semidiurna é a mais importante no local, havendo alguns picos de menor frequência (T 32 e 16 dias).

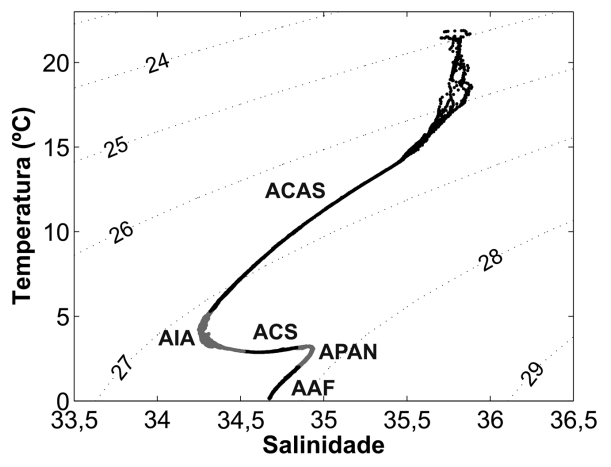

Figura 11 - Diagrama T-S espalhado com identificação das massas d'água presentes no Canal de Vema (foram utilizados os perfis realizados durante a instalação do fundeio VE/338). Para a delimitação de cada massa d'água foram utilizados os critérios de Stramma \& England (1999). 
(1976) sugerem que esse tipo de feição é a assinatura da erosão de camadas mais espessas associadas a velocidades anteriores mais altas (Fig. 12).

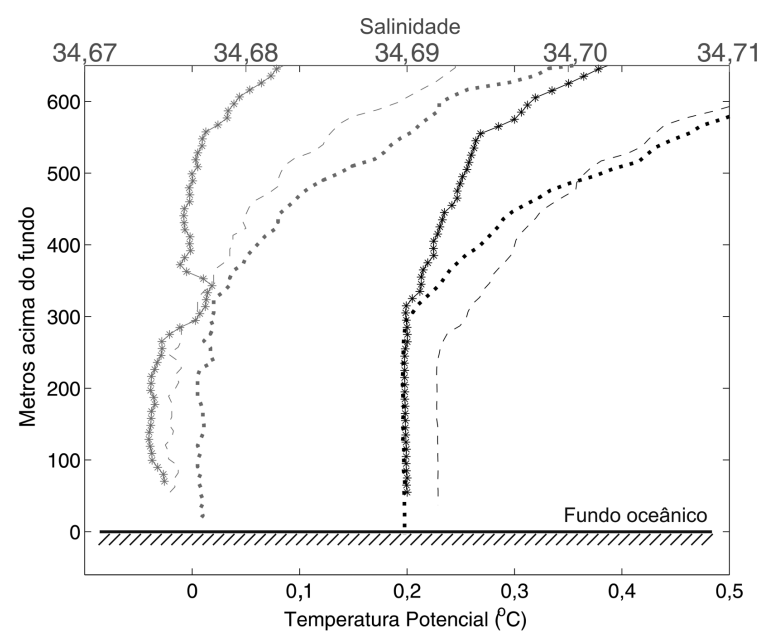

Figura 12 - Salinidade (cinza) e temperatura (preto) da região próxima ao fundo, obtidas a partir de perfis de CTD realizados durante a instalação dos fundeios \#685 (linha tracejada), \#689 (linha pontilhada) e VE/338 (linha com asterisco) mostrando a camada de mistura de fundo (BML).

\section{Material particulado em suspensão}

Perfis de nefelometria realizados em 1971 (ID na Fig. 4) mostram uma espessa camada nefeloide de fundo, com aproximadamente 1000 metros de espessura, abaixo de $3600 \mathrm{~m}$ dentro do canal (Fig. 13). No perfil a jusante (Estação N119) a atenuação da luz foi menos intensa ao longo de todo o perfil e a camada nefeloide de fundo apresentou menor espessura (700 metros), aparecendo apenas a partir de $4000 \mathrm{~m}$ de profundidade. No Canal de Vema a concentração de MPS próximo ao fundo também apresenta variações temporais (Eittreim et al., 1976; Richardson et al., 1987). Cálculos do transporte de material particulado em suspensão (MPS) através do Canal de Vema realizados por Richardson et al. (1987) mostram que o fluxo médio de MPS através do canal é de 6,5 $\times 10^{-4} \mathrm{gs}^{-1}$; entretanto durante os 16 meses de duração dos fundeios analisados por esses autores (1980/81), os valores variaram de 3 a 10,5 × 10-4 gs $^{-1}$ (uma variação de até três vezes). No fundeio \#689 foram observados dois períodos de aumento drástico da concentração de MPS a 80 metros acima do fundo, em janeiro e agosto de 1980. 0 primeiro (janeiro/1980) corresponde a um período de aumento do transporte de AAF e de aumento da velocidade das correntes (Fig. 5); já 0 segundo (agosto/1980) ocorreu quando o transporte de AAF caiu quase a zero e foi observada uma inversão na direção das correntes (Fig. 5), provavelmente relacionada à passagem de um vórtice na região (Hogg et al., 1982; Johnson, 1984) que pode ter causado a ressuspensão local dos sedimentos (Richardson et al., 1987). Nem todos os períodos de altos valores de transporte de AAF e velocidade das correntes corresponderam ao aumento de fluxo de MPS através do canal, e vice-versa.

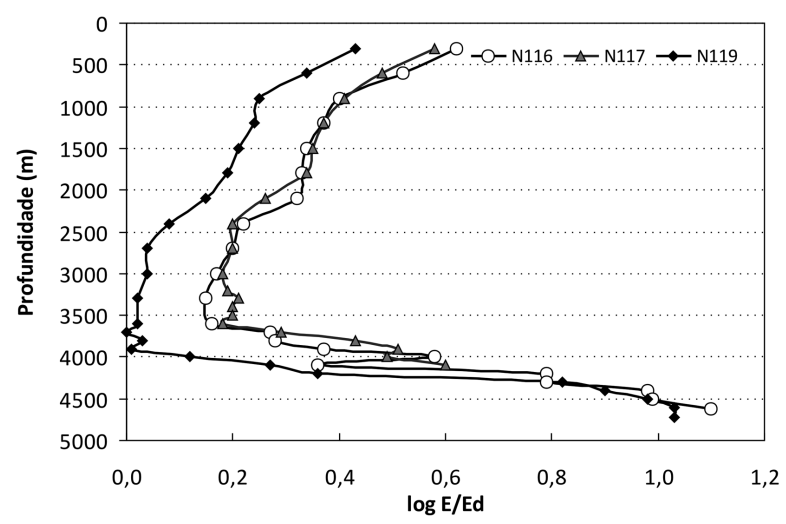

Figura 13 - Perfis de nefelometria em duas estações situadas dentro (N116/N117) e uma estação a jusante (N119) do Canal de Vema. Localização na Figura 4. Dados obtidos em Sullivan et al. (1973). Os valores são expressos como $\log \left(E / E_{D}\right)$, onde $E_{D}$ é a medição de exposição da câmera à luz direta e $E$ é a medição do espalhamento da luz à frente da câmera (Biscaye \& Eittreim, 1977).

Gardner et al. (1997) analisaram amostras de material particulado em suspensão (MPS) coletado em armadilhas de sedimentos colocadas a 53 maf e 94 maf nos fundeios \#685 e \#689, respectivamente; foram encontrados teores de carbonato de cálcio de $11-23 \%$ nas amostras coletadas dentro da BML, os menores valores em toda a coluna d'água. Os mesmos autores também observaram um aumento da concentração de MPS em direção ao fundo, com as maiores concentrações junto à parede leste do canal. Altas concentrações de material particulado em suspensão também foram encontradas em três amostras de água coletadas dentro do canal em 2009 (Tab. 4). Esses valores são maiores do que os anteriormente estimados por Gardner et al. (1997) com base em perfis de nefelometria.

Tabela 4 - Concentração de material particulado em suspensão total (MPS), matéria orgânica total (MOT) e percentual de matéria orgânica nas amostras de água coletadas em outubro de 2009 em três profundidades no Canal de Vema. Localização na Figura 4 (círculo amarelo). Profundidade local: $4800 \mathrm{~m}$. As amostras de água foram filtradas através de filtros de fibra de vidro com tamanho de poro de $0,7 \mu \mathrm{m}$, pré-combustionados a $500^{\circ} \mathrm{C}$ e pré-pesados.

\begin{tabular}{|c|c|c|c|}
\hline Profundidade $(\mathrm{m})$ & [MPS] (mg/L) & [MOT] $(\mathrm{mg} / \mathrm{L})$ & $\%$ de M0 \\
\hline 4236 & 2,96 & 0,68 & 22,8 \\
4286 & 2,51 & 0,67 & 26,8 \\
4316 & 3,55 & 0,63 & 17,7 \\
\hline
\end{tabular}

Segundo Richardson et al. (1987) a fonte principal de MPS para o Canal de Vema são os drifts sedimentares da Bacia da 

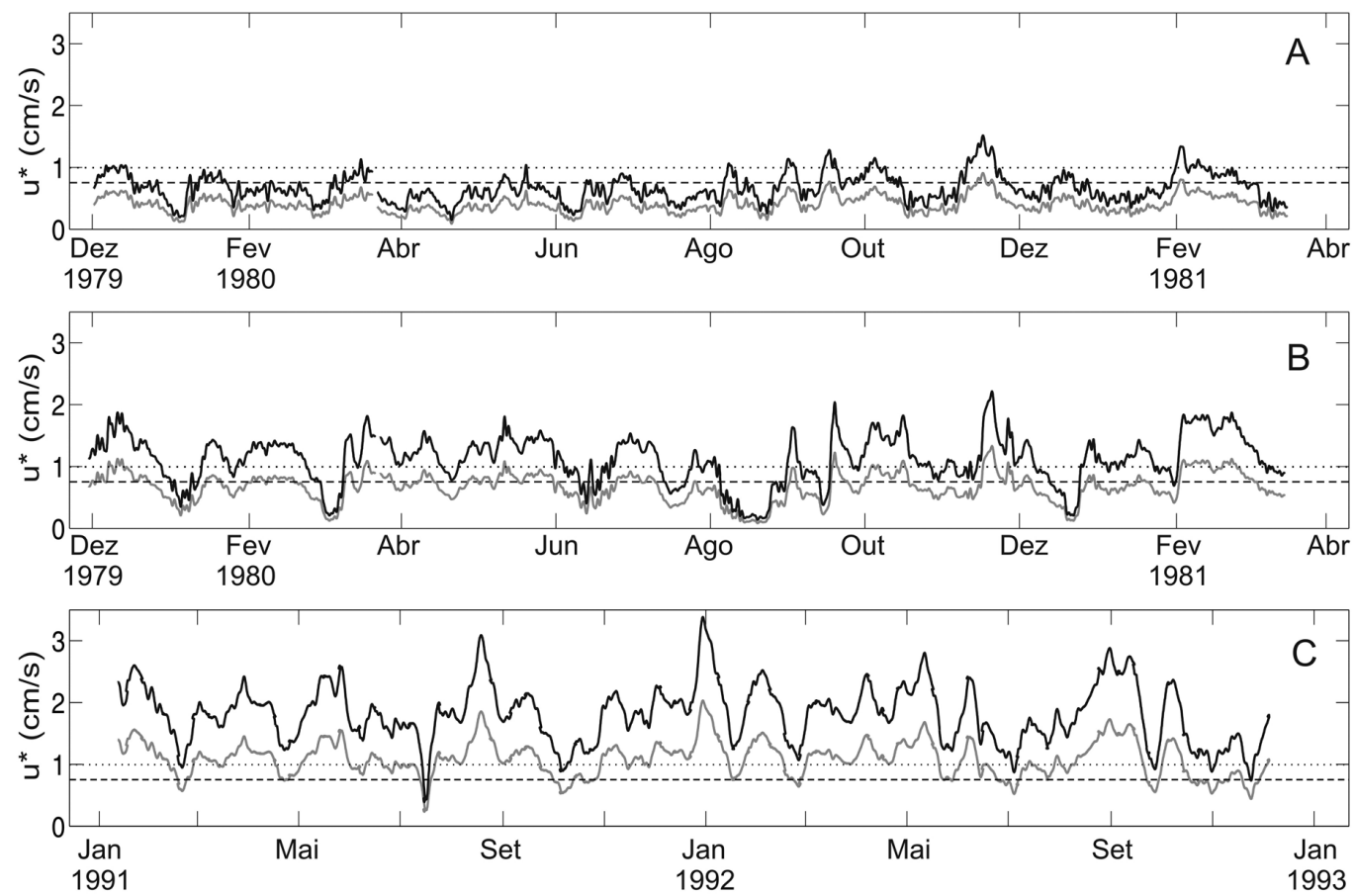

Figura 14 - Velocidades de cisalhamento alcançadas pelas correntes documentadas no Canal de Vema no (A) fundeio \#689; (B) fundeio VE/338 e (C) fundeio \#685 estimadas segundo Bird et al. (1982) (linhas cinzas e pretas representam, respectivamente, os limites mínimos e máximos). As linhas tracejada e pontilhada indicam as velocidades críticas necessárias para iniciar o transporte de silte médio e areia muito fina, respectivamente, com densidade semelhante à do quartzo.

Argentina, uma vez que as incrustações de manganês e o fundo bastante duro sugerem que o fundo do canal não é uma fonte provável de sedimentos, entretanto em situações de correntes muito intensas ou da passagem de vórtices pode ocorrer erosão de sedimentos dentro do próprio canal.

\section{Capacidade de transporte}

A velocidade de fricção média junto ao fundo estimada para 0 fundeio \#689 variou entre 0,7 e 1,1 cm/s, e o valor máximo esteve entre 1,6 e 2,6 cm/s; no fundeio \#685 a velocidade de fricção junto ao fundo não atingiu valores maiores do que $1,9 \mathrm{~cm} / \mathrm{s}$ e a média variou entre 0,4 e $0,7 \mathrm{~cm} / \mathrm{s}$. As maiores velocidades de fricção junto ao fundo foram encontradas no fundeio $\mathrm{VE} / 338$, onde os valores máximos variaram entre 2,0 e $3,4 \mathrm{~cm} / \mathrm{s}$ e os valores médios entre 1,1 e 1,8 cm/s.

Essas estimativas das velocidades de friç̧ão $\left(u^{*}\right)$ mostram que, em geral, as correntes são capazes de suspender e transportar sedimentos de diversos tamanhos granulométricos (Fig. 14). No fundeio \#689 as correntes permaneceram acima da velocidade crítica para transporte de silte médio com densidade $2650 \mathrm{~kg} / \mathrm{m}^{3}$ (semelhante à do quartzo) durante 38 e $82 \%$ do tempo, respectivamente, $u^{*}=0,03 \mathrm{UG}$ e $u^{*}=0,05 \mathrm{UG}$ (Eq. 9); já no fundeio $\mathrm{VE} / 338$, em pelo menos $87 \%$ do tempo $\left(u^{*}=\right.$ 0,03UG; Eq. 9) houve condições para transporte desse tipo de sedimento, havendo alguns eventos de redução das velocidades, quando poderia ocorrer a deposição desses sedimentos. No fundeio \#685, as condições favoráveis ao transporte sedimentar persistiram somente durante 2\% ( $u^{*}=0,03 \mathrm{UG}$; Eq. 9) e $35 \%$ ( $u^{*}=0,05$ UG; Eq. 9) da duração do fundeio. É observada uma redução da capacidade de transporte das correntes ao longo do canal, provavelmente devido ao seu alargamento a jusante, 0 que reduz o confinamento das correntes.

\section{CONCLUSÕES}

0 uso de modelos numéricos de circulação geral é a principal alternativa para o estudo da dinâmica em regiões abissais e também do transporte de sedimentos e da mistura vertical associados a essa dinâmica. Como constatado por Wadley \& Bigg (1996), limitações quanto à resolução e abrangência espacial desses modelos e à inacurácia na representação dos gradientes de densidade transversais às correntes no Canal de Vema, ainda são a maior fonte de dificuldade na aplicação dos modelos numéricos. Esses gradientes, em particular, determinam a estrutura vertical das velocidades geostróficas no interior do canal.

Estimativas do transporte de volume da AAF (4 Sv) no Canal de Vema obtidas pelos estudos numéricos de Wadley \& Bigg (1996), que representaram 0 canal como uma única célula no 
modelo, e o de Maltrud \& McClean (2005), que utilizaram uma resolução espacial (célula) de 1/10 ("eddy resolving"), são condizentes com os valores estimados por Hogg et al. (1999) e Speer \& Zenk (1993) e revelam que a alta resolução não parece ser determinante para esse tipo de cálculo. Entretanto, a simulação dinâmica dos vórtices e filamentos que migram para o canal, bem como do fluxo a jusante e montante, além da compreensão da geração da espessa camada de mistura (BML) observada em seu interior, dependem fundamentalmente de uma adequada representação das feições topográficas locais como discutido por Ezer (2006). A formação das camadas de Ekman de fundo, que induzem uma circulação secundária caracterizada por ressurgência na porção oeste e subsidência na porção leste do canal, descrita no estudo numérico de Jungclaus \& Vanicek (1999), reforça também a já conhecida necessidade de uma melhor parametrização dos coeficientes de fricção turbulenta no fundo, uma das grandes limitações existentes, sobretudo para a modelagem numérica de correntes abissais.

A despeito das dificuldades ainda encontradas, 0 constante aprimoramento de modelos numéricos que utilizam coordenadas verticais seguidoras de terreno e assimilação de diferentes bases de dados tais como altimétricos e de boias de deriva do tipo Argo, fornecem boas perspectivas para a obtenção de representações mais adequadas dos gradientes de pressão barotrópico e baroclíno que controlam a circulação das diversas massas de água no interior do canal.

Os resultados sugerem que o confinamento produzido pelo Canal de Vema intensifica as correntes de fundo e induz à formação de uma camada de mistura de fundo bastante espessa. A informação disponível indica que as condições dinâmicas são favoráveis a altas taxas de transporte sedimentar dentro do Canal, o que é ratificado pelos perfis nefelométricos e as concentrações de MPS total obtidas através da filtração de amostras de água. 0 alargamento do canal leva à redução da intensidade das correntes a jusante e, consequentemente, das taxas de transporte de sedimentos em suspensão. Esses resultados apontam para a possibilidade de que um depósito de deriva ainda pode estar em construção a jusante da boca do canal, onde um leque contornítico vem sendo construído desde o final do Oligoceno (Mézerais et al., 1993).

\section{AGRADECIMENTOS}

Agradecimentos à empresa Repsol YPF S.A. e à Fundação Euclides da Cunha pelas bolsas de Iniciação Cientííica e financiamento concedido a este projeto. Agradecimentos ao Dr. Nelson Hogg (Universidade de Cornell) pelo acesso aos dados de 1980/81.

\section{REFERÊNCIAS}

ARHAN M, CARTON X, PIOLA A \& ZENK W. 2002. Deep lenses of circumpolar water in the Argentine Basin. J. Geophys. Res., 107(C1), 3007. doi: 10.1029/2001JC000963.

ARMI L \& MILLARD RC. 1976. The Bottom Boundary Layer of the Deep Ocean. J. Geophys. Res., 81: 4983-4990.

BARKER PF, JOHNSON DA, CARLSON RL, CEPEK P, COULBOURN WT, GAMBOA LA, HAMILTON N, DE MELO U, PUJOL C, SHOR AN, SUZYUMOV AE, TJALSMA LRC, WALTON WH \& WHALEN E. 1983. Site 518; West Flank, Rio Grande Rise. In: Initial reports of the Deep Sea Drilling Project 72. Texas A \& M University, Ocean Drilling Program, College Station, TX, United States. p. 357-380.

BEAULIEU S \& BALDWIN R. 1998. Temporal variability in currents and the benthic boundary layer at an abyssal station off central California. Deep-Sea Res. II, 45: 587-615.

BIRD AA, WEATHERLY GL \& WINBUSH M. 1982. A Study of the Bottom Boundary Layer over the Eastward Scarp of the Bermuda Rise. J. Geophys. Res., 87: 7941-7954.

BISCAYE PE \& EITTREIM SL. 1977. Suspended particulate loads and transports in the nepheloid layer of the abyssal Atlantic Ocean. Mar. Geol., 23: 155-172.

CUSHMAN-ROISIN B \& BECKERS J-M. 2007. Introduction to Geophysical Fluid Dynamics. Academic Press, 759 pp.

EITTREIM S, THORNDIKE EM \& SULLIVAN L. 1976. Turbidity distribution in the Atlantic Ocean. Deep-Sea Res., 23: 1115-1127.

EZER T. 2006. Topographic influence on overflow dynamics: Idealized numerical simulations and the Faroe Bank Channel overflow. Journal of Geophysical Research, 111, C02002, doi: 10.1029/2005JC003195.

GARDNER WD, BISCAYE PE \& RICHARDSON MJ. 1997. A sediment trap experiment in the Vema Channel to evaluate the effect of horizontal particle fluxes on measured vertical fluxes. J. Mar. Res., 55: 995-1028.

GEBCO - General Bathymetric Chart of the Oceans. 2008. Disponível em: <https://www.bodc.ac.uk/data/online_delivery/gebco/>. Acesso em: 25 fev. 2009.

GUST G. 1989. The benthic boundary layer. In: HELLWEGE KH \& MADELUNG $O$ (Eds.). Landolt-Börnstein numerical data and function relationships, 3b, Oceanography. Springer-Verlag, Berlin, p. 345-398.

HOGG NG \& ZENK W. 1997. Long-period changes in the bottom water flowing through Vema Channel. J. Geophys. Res., 102(C7): 1563915646.

HOGG NG, BISCAYE P, GARDNER W \& SCHMITZ WJ. 1982. On the transport and modification of Antarctic Bottom Water in the Vema Channel. J. Mar. Res., 40(Suppl.): 231-263.

HOGG NG, OWENS WB, SIEDLER G \& ZENK W. 1996. Circulation in the deep Brazil Basin. In: WEFER G, BERGER WH, SIEDLER G \& WEBB D 
(Eds.). The South Atlantic: present and past circulation. Springer-Verlag, 249-260.

HOGG NG, SIEDLER G \& ZENK W. 1999. Circulation and variability at the southern boundary of the Brazil Basin. J. Phys. Oceanogr., 29: 145-157.

HOLLISTER CD \& NOWELL ARM. 1991. HEBBLE epilogue. Mar. Geol., 99: $445-460$.

JOHNSON DA. 1984. The Vema Channel: physiography, structure, and sediment-current interactions. Mar. Geol., 58: 1-34.

JOHNSON DA, McDOWELL SE, SULLIVAN LG \& BISCAYE PE. 1976. Abyssal Hydrography, Nephelometry, Currents, and Benthic Boundary Layer Structure in the Vema Channel. J. Geophys. Res., 81(33): 57715786.

JOHNSON DA, LEDBETTER MT \& BURCKLE LH. 1977. Vema Channel paleoceanography: Pleistocene dissolution cycles and episodic bottom water flow. Mar. Geol., 23: 1-33.

JUNGCLAUS JH \& VANICEK M. 1999. Frictionally modified flow in a deep ocean channel: Application to the Vema Channel. Journal of Geophysical Research, 104(C9): 21123-21136.

KENNETT J. 1982. Marine Geology. Englewood Cliffs: Prentice-Hall, $813 \mathrm{pp}$.

KNUTZ PC. 2008. Paleoceanographic significance of contourite drifts. In: REBESCO M \& CAMERLENGHI A (Eds.). Contourites. Developments in Sedimentology, 60: 511-535.

LE PICHON X, EWING M \& TRUNCHAN M. 1971. Sediment transport and distribution in the Argentine Basin, 2, Antarctic Bottom Current passage into the Brazil Basin. In: AHRENS LH, PRESS F, RUNCORN SK \& UREY HC (Eds.). Physics and Chemistry of the Earth, 8, Pergamon Press, Oxford, p. 31-48.

LEDBETTER MT. 1979. Fluctuations of Antarctic Bottom Water velocity in the Vema Channel during the last 160,000 years. Mar. Geol., 33: 71-89.

LEDBETTER MT. 1984. Bottom-current speed in the Vema Channel recorded by particle size of sediment fine fraction. Mar. Geol., 58: 137149.

LEDBETTER MT \& JOHNSON DA. 1976. Increased transport of Antarctic Bottom Water in the Vema Channel during the last ice age. Science, 194: 837-839.

LI MZ \& AMOS CL. 2001. SEDTRANS96: the upgraded and better calibrated sediment-transport model for continental shelves. Comput. Geosci., 27: 619-645.

LUECK R. 2001. Turbulence in the benthic boundary layer. In: STEELE JH, THORPE SA \& TUREKIAN KK (Eds.). Encyclopedia of Ocean Sciences. Academic Press, San Diego, Vol. 6, p. 3057-3063.

LUKAS R, SANTIAGO-MANDUJANO F, BINGHAM $F$ \& MANTYLA A. 2001. Cold bottom water events observed in the Hawaii Ocean timeseries: implications for vertical mixing. Deep-Sea Res. I, 48: 995-1021.
MALTRUD ME \& McCLEAN JL. 2005. An eddy resolving global 1/10 ocean simulation. Ocean Modelling, 8(1-2): 31-54. doi: 10.1016/j.ocemod.2003.12.001.

MCCAVE IN. 2008. Size sorting during transport and deposition of fine sediments: Sortable silt and flow speed. In: REBESCO M \& CAMERLENGHI A (Eds.). Contourites. Developments in Sedimentology, 60: $121-142$.

MCCAVE IN \& SWIFT SA. 1976. A physical model for the rate of deposition of fine-grained sediment in the deep sea. Geol. Soc. Am. Bull. 87: 541-546.

MCCAVE IN, MANIGHETTI B \& ROBINSON SG. 1995. Sortable silt and fine sediment size/composition slicing: Parameters for palaeocurrent speed and palaeoceanography. Paleoceanography, 10(3): 593-610.

McDONAGH EL, ARHAN M \& HEYWOOD KJ. 2002. On the circulation of bottom water in the region of the Vema Channel. Deep-Sea Res. Part I., 49: 1119-1139.

MELGUEN M \& THIEDE J. 1974. Facies distribution and dissolution depths of surface sediment components from Vema Channel and Rio Grande Rise (Southwest Atlantic Ocean). Mar. Geol., 17(5): 341-353.

MÉZERAIS ML, FAUGÈRES JC, FIGUEIREDO AG \& MASSÉ L. 1993. Contour current accumulation off the Vema Channel mouth, southern Brazil Basin: pattern of a contourite fan. Sediment. Geol., 82(1-4): 173187.

MILLER MC, MCCAVE IN \& KOMAR PD. 1977. Threshold of sediment motion under unidirectional currents. Sedimentology, 24: 507-527.

MOROZOV EG, DEMIDOV AN \& TARAKANOV RY. 2008. Transport of Antarctic waters in the deep channels of the Atlantic Ocean, Dokl. Earth Sci., 423(1): 1286-1289, doi: 0.1134/S1028334X08080230.

NEUMEIER U, FERRARIN C, AMOS CL, UMGIESSER G \& LI MZ. 2008. Sedtrans05: An improved sediment-transport model for continental shelves and coastal waters with a new algorithm for cohesive sediments. Comput. Geosci., 34: 1223-1242.

NOWELL ARM \& HOLLISTER CD. 1985. The objectives and rationale of HEBBLE. Mar. Geol., 66: 1-11.

REBESCO M. 2005. Contourites. In: SELLEY RC, COCKS LRM \& PLIMER IR (Eds.). Encyclopedia of Geology. Elsevier, Oxford, Vol. 4. p. $513-527$.

REBESCO M \& STOW D. 2001. Seismic expression of contourites and related deposits: a preface. Mar. Geophys. Res., 22: 303-308.

RICHARDSON MJ, BISCAYE PE, GARDNER WD \& HOGG NG. 1987. Suspended Particulate Matter Transport through the Vema Channel. Mar. Geol., 77: 171-184.

SALON S, CRISE A \& VAN LOON AJ. 2008. Dynamics of the bottom boundary layer. In: REBESCO M \& CAMERLENGHI A (Eds.). Contourites. Developments in Sedimentology, 60: 83-98.

SCHMITZ WJ \& HOGG NG. 1983. Exploratory observations of abyssal currents in the South Atlantic near Vema Channel. J. Mar. Res., 41: 487510. 
SMITH WHF \& SANDWELL DT. 1997. Global sea floor topography from satellite altimetry and ship depth soundings. Science, 277(5334): 19561962.

SPEER K \& ZENK W. 1993. The flow of Antarctic Bottom Water into the Brazil Basin. Journal of Physical Oceanography, 23: 2667-2682.

STAHR FR \& SANFORD TB. 1999. Transport and bottom boundary layer observations of the North Atlantic Deep Western Boundary Current at the Blake Outer Ridge. Deep Sea Res. Part II, 46: 205-243.

STRAMMA L \& ENGLAND M. 1999. On the water masses and mean circulation of the South Atlantic Ocean. Journal of Geophysical Research, 104(C9): 20863-20883.

SULLIVAN L, THORNDIKE E, EWING M \& EITTREIM S. 1973. Nephelometer measurements, Hach turbidimeter measurements and bottom photographs from Conrad Cruise 15. Lamont-Doherty Geological Observatory, Technical Report No.8-CU-8-73, 259 pp.

VIANA A. 2008. Economic relevance of contourites. In: REBESCO M \& CAMERLENGHI A (Eds.). Contourites. Developments in Sedimentology, 60: 493-510.

VIANA AR, ALMEIDA JR W, NUNES MCV \& BULHÕES EM. 2007. The economic importance of contourites. In: VIANA AR \& REBESCO M (Eds.).
Economic and Palaeoceanographic Significance of Contourite Deposits. Geol. Soc. London Spec. Publ., 276: 1-23.

WADLEY MR \& BIGG GR. 1996. Abyssal channel flow in ocean general circulation models with application to the Vema Channel. Journal of Physical Oceanography, 26(1): 38-48.

ZENK W. 2008. Temperature fluctuations and current shear in Antarctic Bottom Water at the Vema Sill. Progr. Oceanogr., 77: 276-284.

ZENK W \& MOROZOV E. 2007. Decadal warming of the coldest Antarctic Bottom Water flow through the Vema Channel. Geophys. Res. Lett., 34: L14607, doi: 10.1029/2007GL030340.

WHOI - Woods Hole Oceanographic Institute. 2010. Buoy Group Time Series. Disponível em:

$<$ http://gis1server.whoi.edu/website/BGTS/viewer.htm>. Acesso em: 25 jan. 2010.

WOCE - World Ocean Circulation Experiment. 2009. Global Data Resource Version 3.0. Disponível em:

$<$ http://woce.nodc.noaa.gov/woce_v3/wocedata_1/index.htm>. Acesso em: 16 jun. 2009.

\section{NOTAS SOBRE OS AUTORES}

Aline Olivas Kaji é bacharel em Oceanografia pela Universidade do Estado do Rio de Janeiro - UERJ (2010) e mestranda em Engenharia e Gerenciamento Costeiro e Marinho pela Norwegian University of Science and Technology (NTNU). Tem experiência na área de Oceanografia Geológica, com ênfase em dinâmica sedimentar. Suas áreas de interesse são processos sedimentares de áreas costeiras e marinhas, distribuição de material particulado em suspensão, camadas nefeloides.

Josefa Varela Guerra é bacharel em Geografia pela Universidade de São Paulo e Oceanologia pela Fundação Universidade Federal do Rio Grande, mestre em Geografia pela Universidade Federal do Rio de Janeiro e doutora em Oceanografia pela University of Washington (2004). Atualmente é professora adjunta da Faculdade de Oceanografia da Universidade do Estado do Rio de Janeiro. Seus principais temas de interesse relacionam-se à dinâmica sedimentar de ambientes costeiros e oceânicos, aos processos envolvidos nas trocas de material particulado entre as áreas continentais e oceânicas e à aplicação e aperfeiçoamento de técnicas de estudo do transporte de sedimentos.

Alexandre Macedo Fernandes é bacharel em Física pela Universidade Federal do Rio de Janeiro (1998), concluiu mestrado em Oceanografia Física pela Universidade de São Paulo (2001), e doutorado em Oceanografia Física - Florida State University, EUA (2007). Tem trabalhado na área de Oceanografia Física, com ênfase em Variáveis Físicas da Água do Mar e Movimento da Água do Mar, atuando, principalmente, em estudos hidrodinâmicos de pequena à mesoescala, e em estudos de processos de mistura vertical oceânicos. Atualmente é professor adjunto da Faculdade de Oceanografia da Universidade do Estado do Rio de Janeiro atuando no Departamento de Oceanografia Física.

Roberto Freires de Oliveira é bacharel em Oceanografia pela Universidade do Estado do Rio de Janeiro - UERJ (2011) e mestrando em Geologia e Geofísica Marinha pela Universidade Federal Fluminense. Tem experiência na área de Oceanografia Geológica, com ênfase na dinâmica sedimentar de ambientes costeiros e marinhos. Atualmente trabalha com processos sedimentares e evolução de depósitos de mar ultra-profundo nas Bacias de Pelotas e Santos, distribuição de material particulado em suspensão e identificação de camadas nefeloides no Atlântico Sul.

Cleverson Guizan Silva é bacharel em Geologia pela Universidade Federal do Rio de Janeiro - UFRJ (1982), mestre em Geologia pela Universidade Federal do Rio de Janeiro (1987) e doutor em Geologia pela Duke University, EUA (1991). Atualmente é professor associado da Universidade Federal Fluminense - UFF. Seus temas de interesse inserem-se em geologia e geofísica marinha, como sistemas deposicionais marinhos e costeiros, tectônica de argilas, hidratos de gás e instabilidades do talude, diagnóstico e monitoramento ambiental em áreas marinhas e costeiras.

Antonio Tadeu dos Reis é bacharel em Geologia pela Universidade Federal do Rio de Janeiro - UFRJ (1985), mestre em Geofísica pelo Observatório Nacional - CNPa (1994) e doutor em Análise de Bacias pela Université Pierre et Marie Curie - Paris VI, França (2001). Atualmente é professor adjunto da Faculdade de Oceanografia - UERJ. Suas áreas de interesse são processos gravitacionais (tectônica e transporte de massa), análise de bacias, e ciclicidade e arquitetura sedimentar de sistemas deposicionais marinhos. 